\title{
The Effect of Regional Climate Model Domain Choice on the Simulation of Tropical Cyclone-Like Vortices in the Southwestern Indian Ocean
}

\author{
Willem A. Landman, Anji Seth, and Suzana J. Camargo \\ International Research Institute for Climate Prediction, Lamont-Doherty Earth Observatory, Columbia University, \\ Palisades, New York
}

(Manuscript received 18 December 2002, in final form 30 September 2004)

\begin{abstract}
A regional climate model is tested for several domain configurations over the southwestern Indian Ocean to examine the ability of the model to reproduce observed cyclones and their landfalling tracks. The interaction between large-scale and local terrain forcing of tropical storms approaching and transiting the island landmass of Madagascar makes the southwestern Indian Ocean a unique and interesting study area. In addition, tropical cyclones across the southern Indian Ocean are likely to be significantly affected by the large-scale zonal flow. Therefore, the effects of model domain size and the positioning of its lateral boundaries on the simulation of tropical cyclone-like vortices and their tracks on a seasonal time scale are investigated. Four tropical cyclones, which occurred over the southwestern Indian Ocean in January of the years 1995-97, are studied, and four domains are tested. The regional climate model is driven by atmospheric lateral boundary conditions that are derived from large-scale meteorological analyses. The use of analyzed boundary forcing enables comparison with observed cyclones in these tests. Simulations are performed using a $60-\mathrm{km}$ horizontal resolution and for an extended time integration of about 6 weeks. Results show that the positioning of the eastern boundary of the regional model domain is of major importance in the life cycle of simulated tropical cyclone-like vortices: a vortex entering through the eastern boundary of the regional model is generally well simulated. The size of the domain also has a bearing on the ability of the regional model to simulate vortices in the Mozambique Channel, and the island landmass of Madagascar additionally influences storm tracks. These results show that the regional model can produce cyclonelike vortices and their tracks (with some deficiencies) given analyzed lateral boundary forcing. Statistical analyses of GCM-driven nested model ensemble integrations are now required to further address predictive skill of cyclones in the southwestern Indian Ocean and to test if the model can realistically simulate tropical storm genesis as opposed to advecting existing tropical disturbances entering through the model boundaries.
\end{abstract}

\section{Introduction}

General circulation models (GCMs) have the ability to create tropical vortices that have good similarities with observed tropical cyclones. Indicators such as convergence, high moisture content, and heavy precipitation at the lower levels are skillfully simulated (Vitart and Stockdale 2001). However, the coarse resolution of GCMs results in a lack of the presence of an eye and associated eyewall, and the simulated storms have a larger horizontal extent than those observed (Bengtsson et al. 1995), which has a strong impact on the intensity of the model tropical storms and on their tracks. Although the characteristics of tropical cyclone-like storm statistics in GCMs vary among different models (Camargo and Zebiak 2002a), these tropical storm

Corresponding author address: Dr. Willem A. Landman, South African Weather Service, Private Bag X097, Pretoria 0001, South Africa.

E-mail: willem@weathersa.co.za tracks generated by GCMs (e.g., Vitart et al. 1997) are generally located too far to the east over the southern Indian Ocean. While there are a few select research institutions that have the capacity to run global highresolution simulations to study tropical cyclones, regional and local research institutions, particularly in developing/transitioning economies, do not have such computational capacity at present, and yet there is a pressing need in many of these tropical and subtropical regions to improve regional forecasting capacity using currently viable methods. An alternative, more economical approach to improving model resolution is by nesting a limited-area or regional climate model within a GCM (Giorgi et al. 1993a, b; McGregor 1997). These regional models have been found to produce cyclones that are weaker than those observed but more realistic than the vortices generated by a GCM (Walsh and Watterson 1997; Nguyen and Walsh 2001).

Regional climate model simulations of mesoscale components of the monsoon regions are not strongly sensitive to domain size (Bhaskaran et al. 1996). How- 
ever, synoptic disturbances generated within a midlatitude regional climate model domain are larger in amplitude (geographical size) than those generated within a tropical or subtropical domain and are more likely capable of interacting with and altering the long-wave circulation obtained from the large-scale driving field (Bhaskaran et al. 1996; Jones et al. 1995). Tropical cyclones, classified by some as a mesoscale phenomenon (Holton 2004), have their tracks across the Indian Ocean significantly influenced by the large-scale zonal flow, so much so that the risk of landfall over southeastern Africa significantly increases with a more westward-steering flow (Vitart et al. 2003). These large storms therefore are relatively small-scale phenomena and are at the same time affected by the large-scale circulation. Therefore, it is necessary to investigate the effect that the size of the regional model domain and the positioning of its boundaries have on the simulation of tropical cyclone-like vortex tracks and their landfall, as well as storm intensity within a regional climate model. Increasing the size of the model domain does not always improve simulations, especially over datasparse areas (Bormann and Marks 1999) such as the southwestern Indian Ocean. Therefore careful consideration of domain choice and the location of the lateral boundaries are essential (Seth and Giorgi 1998).

In this paper, a feasibility study is presented for the use of a regional climate model to "downscale" lowresolution atmospheric forcing, a method that many regional research institutions do (or will soon) have the capability to utilize. To investigate the feasibility of using this nested approach, a regional climate model (60$\mathrm{km}$ resolution) is forced along the boundaries of the limited-area domain with time-dependent large-scale meteorological analyses data (about $250-\mathrm{km}$ resolution). The use of analyses data as the boundary forcing will enable an evaluation of the regional model performance given "perfect" boundary forcing. The regional model is used in this paper to investigate the simulation of individual cyclonelike vortices over the southwestern Indian Ocean (e.g., Nguyen and Walsh 2001) as opposed to the use of GCMs (e.g., Vitart et al. 1997, 1999). Here, no attempt is made to simulate the seasonal characteristics of tropical cyclones (e.g., storm frequency and interannual variability). That will require a large number of cases nested in GCM large-scale fields and will be addressed in a later study. Instead, this investigation focuses on whether or not a regional model has the ability to simulate the migration of tropical vortices across the Madagascar island landmass and also to generate storms within the Mozambique Channel. This island with significant topographical features can potentially alter the life cycle of tropical cyclones, since it affects the low-level flow, a notion supported by regional model work over New Guinea (Walsh and Watterson 1997).

Four domains are considered to simulate the characteristics of tropical cyclone-like vortices that occurred during the months of January from 1995 to 1997. The domains are selected in such a way that the southern and western boundaries remain stationary since they have an insignificant effect on tropical phenomena coming from the east, but with varying northern and eastern boundaries. Given a nearly perfectly forced regional model, three aspects of simulation are investigated: first, the effect domain choice has on westwardmigrating simulated tropical cyclone-like vortices over the southwestern Indian Ocean; second, the effect Madagascar has on westward-propagating storms; and third, the ability of the regional model to simulate a tropical cyclone-like vortex inside the Mozambique Channel. If these cases are realistically simulated by the regional model, a nested approach has the potential to add to the predictability of storm tracks over the southwestern Indian Ocean on a seasonal time scale, and it may be worth testing the model nested in a GCM.

\section{Method and data}

\section{a. The regional climate model and domain setup}

The regional model is the National Center for Atmospheric Research (NCAR) Regional Climate Model version 2 (RegCM2; Giorgi et al. 1993a,b), which is a second-generation regional climate modeling system. The dynamical component of the RegCM2 is essentially the same as that of the standard fourth-generation Pennsylvania State University (PSU)-National Center for Atmospheric Research (NCAR) Mesoscale Model (MM4), which is a hydrostatic, compressible, primitive equation, terrain-following $\sigma$ vertical coordinate model, with 14 vertical levels. The physics parameterizations of the RegCM2 are described in Giorgi et al. (1993a,b) and Seth et al. (1999). The cumulus parameterization scheme is that described by Grell (1993). Since the domain is not global, its lateral boundaries require periodic forcing of the model prognostic variables by applied external conditions (Seth et al. 1999). A one-way nesting technique (Giorgi et al. 1994) is utilized whereby European Centre for Medium-Range Weather Forecasts (ECMWF) analyses meteorological fields at T42 resolution (Trenberth 1992) are used as driving initial and time-dependent lateral conditions (6 hourly) for the RegCM2, with sea surface temperatures specified from a dataset of monthly mean values (Reynolds and Smith 1994).

The forcing meteorological fields have a horizontal resolution used by most of the current operational GCMs for dynamical seasonal forecasting (e.g., Barnston et al. 2003), which means that the coarser resolution, as used in GCMs, would therefore not capture the observed scale and intensity of those tropical cyclones prior to entering the domain, since the success of tropical cyclone characteristic simulation depends on a high grid resolution and proper initial vortices (Liu et al. 1997). In addition to resolution, physical parameteriza- 


\section{Domains}

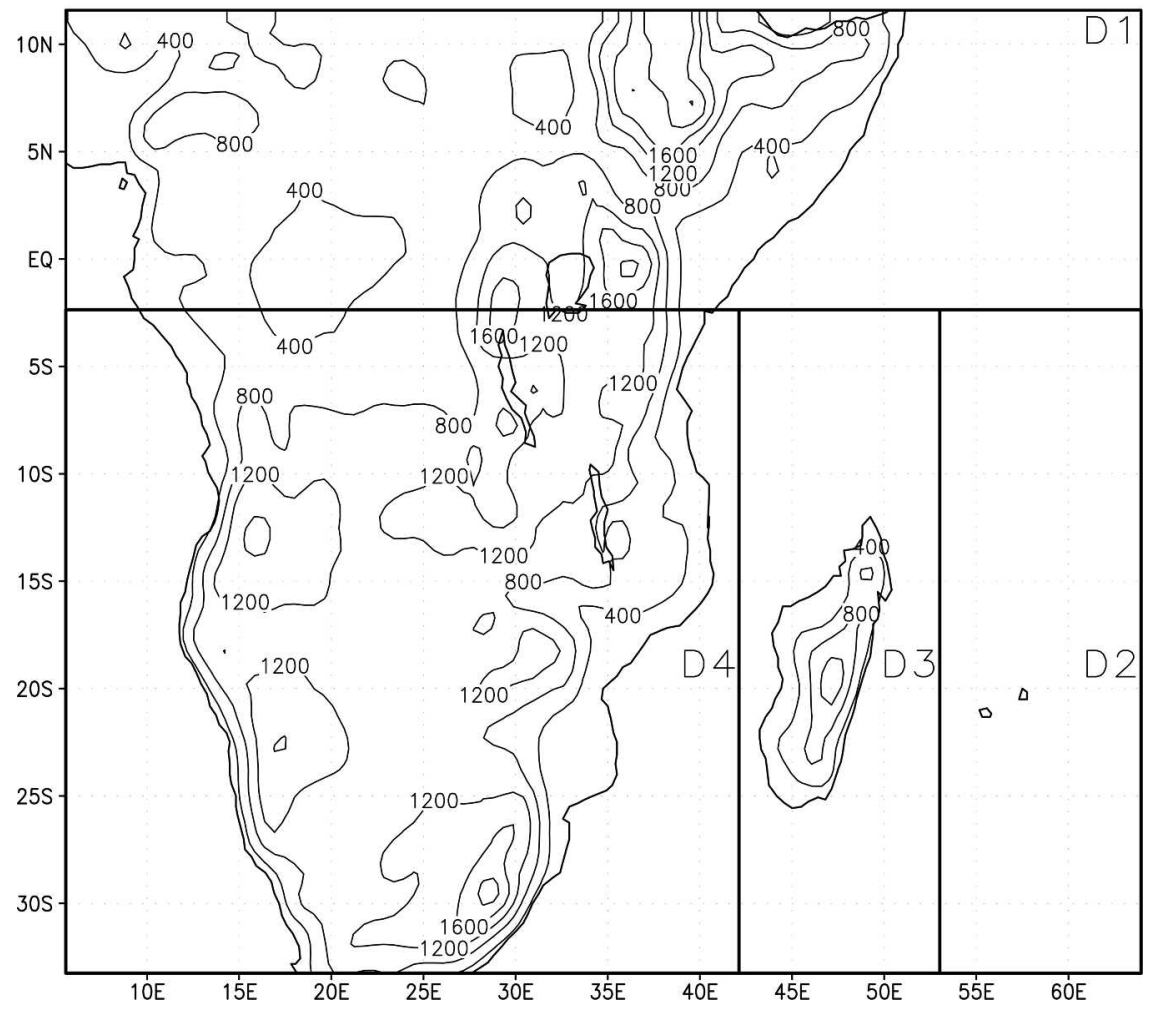

FIG. 1. The four regional model domain configurations (labeled D1 to D4) used in the analyses. Heights contours are in meters.

tion schemes have a significant influence on the simulation of tropical storms (Vitart and Stockdale 2001). No attempt is made to synthetically strengthen the cyclonelike vortex with realistic size, depth, and intensity at the position of entry into the respective domains, or where regional model tropical cyclone-like vortices may happen to develop inside the domain. The use of such a "synthetic" tropical cyclone-like vortex of similar location, strength, and motion to the observed one, referred to as bogusing (Kurihara et al. 1993; Peng et al. 1993; Serrano and Unden 1994; Ueno 1989), requires some foreknowledge of tropical cyclone-like structure and position and is not relevant to the problem of producing skillful predictions of tropical cyclone activity operationally on a seasonal time scale (Walsh 1997). Bogusing is also more likely to improve only on the short-range prediction of tropical cyclone-like vortex characteristics (Serrano and Unden 1994).

Experiments were performed with varying domain sizes in the simulation of tropical cyclone-like vortices for the period 16 December 1995 to 31 January 1996. Initial tests varying the northern boundary $\left(12^{\circ} \mathrm{N}\right.$ to $12^{\circ} \mathrm{S}$ and not presented here) showed that a northern boundary far removed from the storm track of those vortices moving from east to west in the analyses had very little effect on the regional model simulations.
However, a northern boundary too close to the analyses' storm track distorted the simulated circulation patterns significantly. The greatest effect on the simulated life cycle of these tropical cyclone-like vortices is associated with the positioning of the eastern boundary. Based on these initial tests, four main domains were selected for this study and are given in Fig. 1. The first two [domains 1 (D1) and 2 (D2)] have an eastern boundary at about $64^{\circ} \mathrm{E}$, with the eastern boundaries of the remaining domains closing in toward Madagascar [53 ${ }^{\circ} \mathrm{E}$ for domain 3 (D3) and $42^{\circ} \mathrm{E}$ for domain 4 (D4)]. The northern boundaries are, respectively, north $\left(12^{\circ} \mathrm{N}\right)$ for $\mathrm{D} 1$ and south of the equator $\left(3^{\circ} \mathrm{S}\right)$ for $\mathrm{D} 2$, D3, and D4.

The model horizontal resolution is $60 \mathrm{~km}(\mathrm{D} 1: 90 \times$ 111 grid points; D2: $64 \times 111$; D3: $64 \times 91$; D4: $64 \times 71)$ and $40 \mathrm{~km}(\mathrm{D} 2: 94 \times 167)$, and the model is run for both horizontal resolutions at a time step of $150 \mathrm{~s}$. The driving lateral conditions are taken from 6-hourly ECMWF analyses at $\mathrm{T} 42$ resolution and interpolated spatially to the model grid and temporally to the model time step. The boundary forcing is applied in a buffer zone wherein a weighting function equal to 1 at the boundaries decreases exponentially inward (Seth et al. 1999). Within the buffer zone, the forcing is strongest in the upper layers and decreases toward the surface. Easterly 
environmental flow being advected into the eastern boundary may result in a boundary forcing influence extending further inside the nested domain than the buffer zone. The number of grid points in the buffer zone depends on the size of the domain. Here, the buffer zones vary for the domains: D1 and D2 use 14 grid points (about $800 \mathrm{~km}, 7^{\circ}$ ); D3 and D4 use 6 grid points (about $300 \mathrm{~km}, 3^{\circ}$ ). The RegCM2 is initialized on the 16th of the Decembers preceding each of the three Januaries from 1995 to 1997 . Integration is terminated after 50 days, with the first 16 days of simulation discarded to allow for model spinup (Anthes et al. 1989). For tropical cyclone-like vortices, increased horizontal resolution should be used in preference to vertical resolution (Keen and Glenn 1998). Although substantially higher horizontal resolution than the $60 \mathrm{~km}$ used here is required to simulate realistic storm intensities or eye structure, simulations performed on a horizontal resolution of $40 \mathrm{~km}$ on the same domain did not produce any significant improvement in simulation performance, as was also found in regional model simulations over the upper Mississippi basin (Seth and Giorgi 1998).

Error growth is artificially limited for a model forced with observed meteorological fields if a sufficiently small domain is used. Forcing the regional climate model with analyses data, the smaller the domain the better the hindcast simulation (Chouinard et al. 1994). Notwithstanding this issue, the smallest of the four domains is selected for some cases in order to eliminate the effect the model topography of Madagascar has on the simulation of tropical cyclone-like vortex characteristics. A simulation is also run where the model topography has been reduced significantly to further investigate the role topography, especially that of Madagascar, plays on modeled tropical cyclones. Although the smaller two domains are located close to the island, no special treatment of the topography is attempted in order to achieve more skillful simulations (Walsh and McGregor 1995).

\section{b. Tracking the storms}

The simulated cyclonelike vortex tracks are determined by a tracking algorithm (Camargo and Zebiak 2002 b), which determines the position of the storms every $6 \mathrm{~h}$ as the centroid position of the low-level (850 $\mathrm{hPa}$ ) vorticity, analogous to a center of mass, once the existence of the tropical cyclone is previously determined by examining the properties of a vortex with a closed circulation. The threshold vorticity value used in the tracking algorithm is $1.5 \times 10^{-5} \mathrm{~s}^{-1}$. This procedure has been used successfully to track tropical cyclone-like vortices in different GCMs (Camargo and Zebiak 2002b; Camargo and Sobel 2004). The low-level vorticity maximum probably is a sufficient parameter to use over the south Indian Ocean in estimating the simulated tropical cyclone-like vortex position here, once the vortex is identified as a tropical cyclone. In many modeling studies, low-level vorticity is one of the fields used to identify and track tropical cyclone-like vortices. Here, the vortices are first identified visually by a closed cyclonic circulation, and only once this is done can their trajectory be determined by the vorticity. Tropical cyclones in the analyses are tracked in the same way.

After the tracking algorithm has determined the center of each vortex at $850 \mathrm{hPa}$ for each time step, this same location per time step is used at higher atmospheric levels to obtain the upper-air characteristics of the vortex (area-averaged wind speed, temperature, pressure heights, etc.). This calculation is done at different pressure levels, thus assuming that the vertical structure of the vortices remains intact throughout its life cycle. This has been found to be the case for most of the vortices' duration, except toward their demise when the higher levels' closed circulation of the vortex either falls behind or leads the lower-level closed circulation.

The tracking algorithm requires a minimum number of model grid points to be applied. This means that when the tropical cyclone is very near the domain border, the tropical cyclone cannot be tracked by this algorithm, although the simulated winds and other fields indicate its presence close to the boundary. Therefore, some of the figures may create the impression that vortices have originated west of the eastern boundary when in fact they could only be tracked by the algorithm a short number of time steps after entering the domain.

\section{c. Tropical cyclones in the analyses}

ECMWF analyses data do not represent every observed tropical storm (Walsh 1997). This has also been found with ECMWF reanalyses data where the representation of Southern Hemisphere tropical cyclones has a hit rate of $86 \%$ (Serrano 1997). The missing storms are primarily a result of lack of data; most of the missing storms are located far from major landmasses (which is the case with the southwestern Indian Ocean). Since data coverage in the analyses is not optimal, their representation of real tropical cyclones is therefore inherently uncertain; even in data-rich regions, some essential data may be missing, which will have a detrimental effect on the depiction of tropical cyclones in the analyses (Walsh 1997). However, tropical cyclone structure, as represented in the analyses where data coverage is adequate, is similar to reality except for the wind speed structure close to the center of the storms. Further tropical cyclone structure problems are found with small but intense storms, since the analyses underestimate the minimum surface pressure and maximum wind speeds (Walsh 1997). Notwithstanding these problems, it was possible to identify four tropical cyclones in the analyses, and they are presented in this paper.

The identified storms are Bonita (1-15 January 1996; Fig. 2), Christelle (1-10 January 1995; Fig. 4), Fabriola (31 December 1996-9 January 1997; Fig. 6) and Gretelle (19-31 January 1997; Fig. 8). In Figs. 2a, 4a, 6a, and 
8a, the storm tracks of the four cyclones are shown as given by the Joint Typhoon Warning Center (JTWC) data (available online at http://www.npmoc.navy.mil/ jtwc.html; black lines) and as these storms appear in the ECMWF analyses (gray lines). These figures show the first and last days of the storms with the tracks given every $6 \mathrm{~h}$, and each new day is marked with an asterisk. These storms are representative of storms developing east of Madagascar, including those that have landfall as they move across Madagascar and sometimes into southern Africa and those that migrate toward the southeast as they move into the midlatitudes. Close agreement is found between the analyses tracks and that of the JTWC, except for Christelle, where the JTWC data do not identify a tropical vortex for the period 1-3 January 1995 (Fig. 4). Figures are discussed in greater detail in the following section.

Christelle moved across the eastern boundary of the largest domain (Fig. 1) on 1 January 1995 and was back near that boundary on 10 January (Fig. 4a). Bonita moved across the location of the eastern boundary of D1 and D2) on 8 January 1996, made landfall at Madagascar on 11 January, moved across the location of the eastern boundary of D4 between 12 and 13 January, and subsequently made landfall at the subcontinent on 14 January. Soon after, it moved zonally as a tropical depression across the subcontinent where it died at $21^{\circ} \mathrm{E}$ (Fig. 2a). Tracks similar to Bonita's were also observed more recently between 4 and 23 February (Leon-Eline), and 24 March to 9 April 2000 (Hudah). Fabriola also made landfall twice, but once on the east coast of Madagascar, followed by a west coast landfall a few days later. This vortex began on the 30 December 1996 between the location of the eastern boundaries of D2 and 3 D3 and then moved south across the island to die over the ocean on 10 January 1997 (Fig. 6a). Gretelle made landfall once at Madagascar on 24 January 1997, migrated into the channel, and curved counterclockwise until its demise on 31 January. This storm originated between the locations of the eastern boundaries of D2 and D3 (Fig. 8a), as was the case with Fabriola. Of the four tropical cyclones, two moved across the eastern boundary of D1 and D2 as they entered the regional model domain (Christelle and Bonita). Two developed east of the eastern boundary of D3 but west of the eastern boundary of D1 and D2 (Fabriola and Gretelle).

\section{RegCM2 simulation results}

The performance of the regional model is investigated by graphically comparing the track; the 850-, $500-$, and $300-\mathrm{hPa}$ geopotential heights; wind magnitude; temperature; and the $850-200-\mathrm{hPa}$ vertical wind shear of the simulated cyclonelike vortices and the cyclones of the analyses. The heights and temperature values are $5^{\circ} \times 5^{\circ}$ area-averaged values, centered around the calculated storm center. Since it is assumed that the vertical structure of the vortices remains intact throughout its life cycle, values at higher levels are also centered around the geographical location of the 850$\mathrm{hPa}$ storm center. Although tangential storm wind speeds of the analyses are similar to those observed, certain storms are poorly represented (Walsh 1997). In addition, the wind magnitudes shown here are $10^{\circ} \times$ $10^{\circ}$ area-averaged values centered around the calculated storm center, since this area is representative of the horizontal extent of the storms. The area used for calculating the average values for heights and temperature differ from that of wind magnitudes because the heights and temperature are used to represent the storm center and therefore its intensity, while winds are used to represent the horizontal extent of the storm. Both of these factors lead to area-averaged wind magnitudes that are lower than the normally observed maximum wind speed near tropical storm centers.

\section{a. Bonita}

Landfall forecasts for the Atlantic basin have been found to be most accurate for storms moving at oblique or normal angles to the coastline (Powell and Aberson 2001). Although Tropical Cyclone Bonita approached the southern African coast line at an oblique angle (Fig. 2 ), testing the accuracy of storm-track simulations moving at specific angles to the coast line lies outside the scope of this paper since many simulations in the southwestern Indian Ocean are required to test for this. The simulated track using domain configurations of D1 is shown in black and of D2 in gray (Fig. 2b). This cyclone was subject to the enhanced westward-steering flow during the early part of 1996. Simulations using domain configurations D1 and D2 (Fig. 2b) show the tropical cyclone-like vortex moving across Madagascar and the Mozambique Channel and into southern Africa at landfall. The migration of the regional model's cyclonelike vortex into the channel is an indication of the regional model's ability to propagate these vortices sufficiently westward. Moreover, the placement of the eastern boundary in the channel (D4), therefore excluding Madagascar in the regional model run, has the duration of the tropical cyclone-like vortex track (not shown) increased by at least 3 model days over the larger domain runs so that its time of demise corresponds to that of the storm's representation in the analyses. Also, the track produced from using this domain configuration (D4) has as a result the tropical cyclone-like vortex progressing further into the interior as opposed to those simulated using the larger domains. The simulation using D2 with the much reduced topography $(\sim 2 \mathrm{~m}$ at its highest elevation) does not improve on the tropical cyclone-like vortex track since a split vortex is produced northeast of Madagascar (Fig. 2c). One part of the vortex curves on an unrealistic northbound track opposite to the southward track normally observed for tropical cyclones east of Madagascar. The more realistic part of the split storm progresses across the channel as before 
(a)

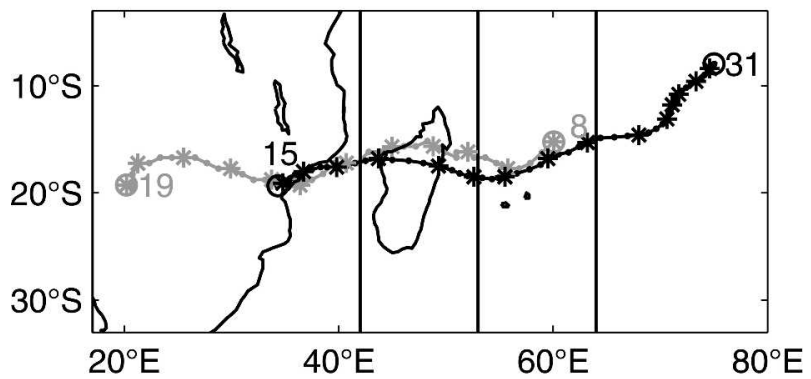

(b)

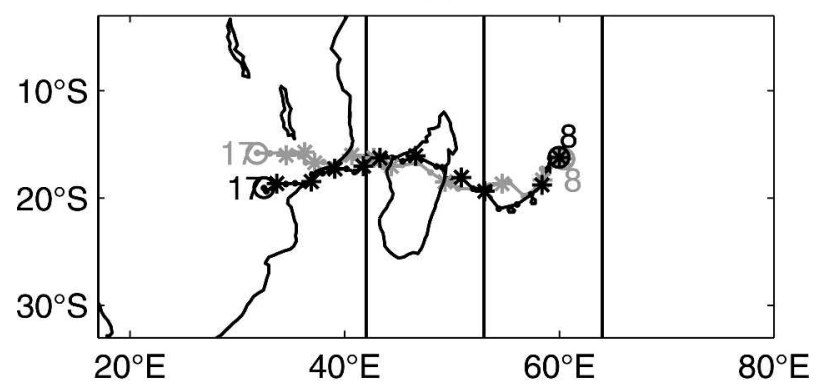

(c)

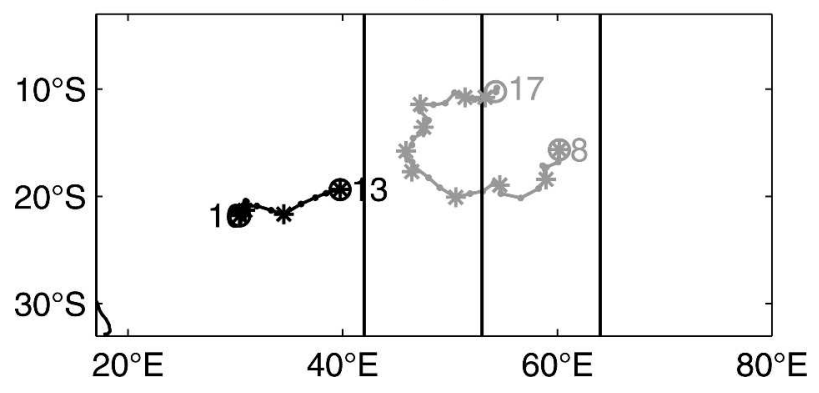

FIG. 2. (a) Observed (JTWC; black line) and ECMWF analyses (gray line) tracks of Tropical Cyclone Bonita (1-15 Jan 1996). (b) Simulated tracks of Bonita using the configurations of D1 (black line) and D2 (gray line). (c) Simulated tracks of Bonita using domain configuration D2 with a maximum topography of $\sim 2 \mathrm{~m}$. A split vortex track is produced on top of Madagascar (gray line: northward-curving track; black line: westward track). The first and last days of the storms are numbered and circled, and the tracks every $6 \mathrm{~h}$ and for each new day are marked with dots and asterisks, respectively.

but farther inland than the vortices of the D1 and D2 simulations with normal model topography. This farther westward progression of the simulated storm is indicative of the influence Madagascar has on the momentum of low-level flow, because the reduced model topography did not slow the storm down as much as was found in the simulations with a more realistic topography.

Figure 3 shows the comparison between the simulated (thin numbered lines) and analyses (thick line) tropical cyclone-like vortex 850-, 500-, and 300-hPa geopotential heights (again, spatial averages around the defined storm center) of the vortex supplied to the eastern boundary of the four domains. The thin-line num- bers correspond to the domain numbers. The left-hand panels correspond to domains D1 and D4, while the right-hand panels show the results of the simulations using domains D2 and D3. While the model simulates an intensification of the vortex over the 2-day period from 10 to 12 January, the intensification is weaker than shown in the analyses. Moreover, as soon as the simulated vortex moves outside of the buffer zone, the model's lower and middle geopotential heights at the storm's center deviate from that of the analyses' intensification. However, the modeled vortex heights at middle to high atmospheric levels compare well with analyses, with the best simulations made for the 300hPa level (Fig. 3). Similar results are found for the modeled winds (not shown). Also found is that the modeled low-level temperature (not shown) is constantly below that of the analyses, which will lead to less favorable conditions for deep convection, and subsequently to the modeled vortex being of lesser intensity than the analyses near the surface.

The smallest of the domains (D4) has its eastern boundary west of Madagascar, resulting in much farther westward migration of the vortex into the subcontinent (not shown) as is found using the other domain configurations. This is mainly due to the regional climate model eastern boundary being close to the area of interest, which will subsequently limit error growth artificially (Chouinard et al. 1994). The simulation with the D4 configuration illustrates the effect the close proximity of the boundary has on simulated tropical cyclone-like vortex tracks. To further illustrate this effect, the evolution of Tropical Cyclone Christelle during January 1995 is subsequently presented.

\section{b. Christelle}

Tropical Cyclone Christelle moved into D1 of the regional model on 1 January 1995 (Fig. 4). It began in a southwesterly direction and moved southeastward as it moved out of the domain on 10 January. Its entire track is not more than $10^{\circ}$ of longitude away from the eastern boundary of D1 and D2, which is at a distance of about $6^{\circ}$ west of the eastern boundary of the domain (see also section 2a). The modeled tropical cyclone-like vortex (Fig. 4b) stayed even closer to the eastern boundary of the domain than the analyses storm but moved away from the buffer zone on 5 January. Figure 5 shows the geopotential heights of Cyclone Christelle of the analyses data and the simulations done with domain configurations D1 and D2. There is close agreement between the low-level simulated vortex and analyses storm geopotential heights (averaged around the calculated storm center) until it moves away from the buffer zone on 5 January. Thereafter, the modeled vortex at the 850-hPa level has a lower intensity than the analyses storm, except during the latter part of the D1 simulation, where simulated intensities are more similar to those observed as a result of the storm track in D1 once again approaching the boundary of the domain. This 

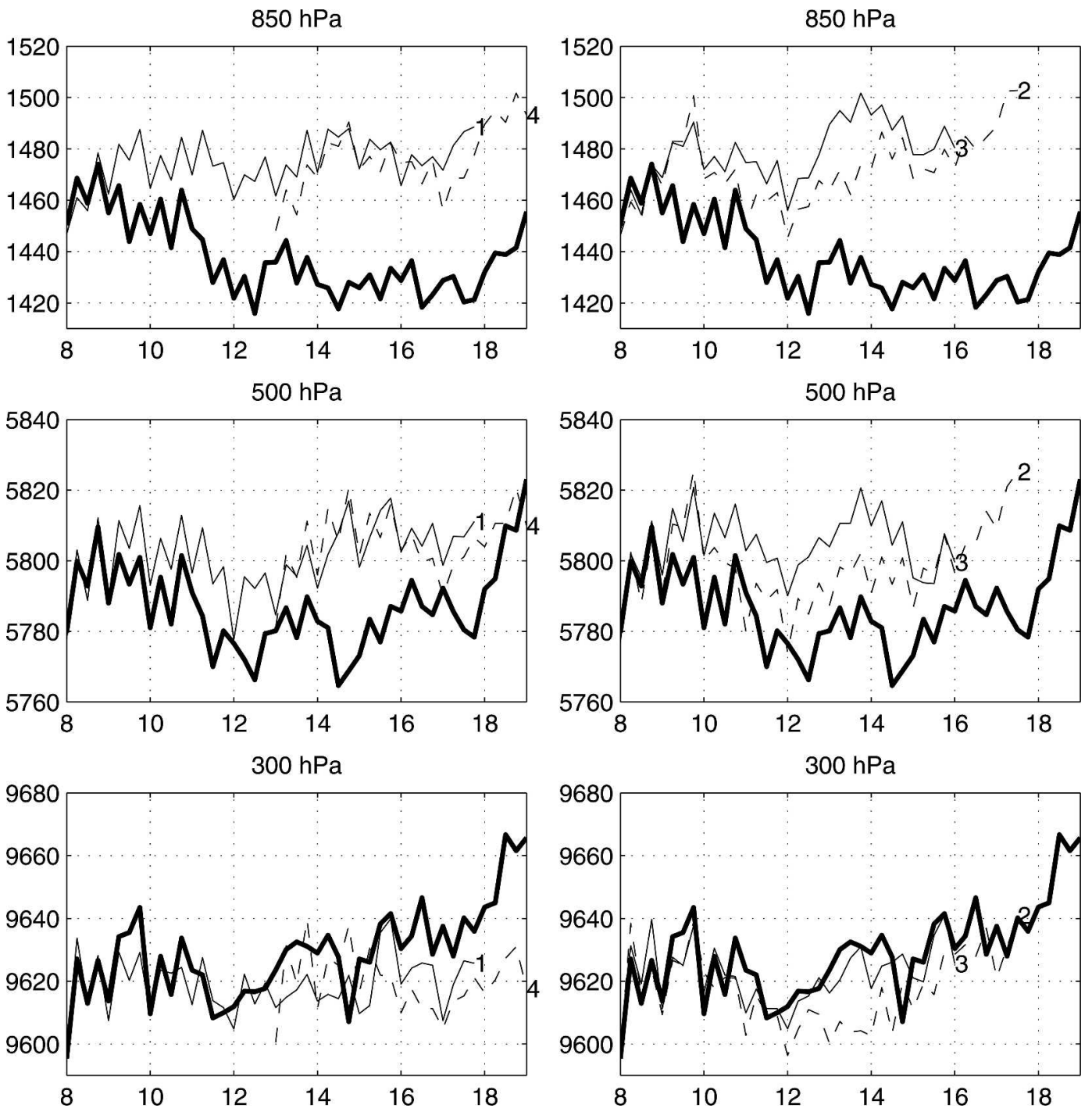

FIG. 3. Geopotential heights at 850,500 , and $300 \mathrm{hPa}$ of the analyses (thick line) and simulations (numbered thin lines) of Bonita for each of the four domain configurations. The thin-line numbers correspond to the domain numbers. The left-hand panels correspond to domains D1 (thin solid) and D4 (dashed), while the right-hand panels show the results of the simulations using domains D2 (dashed) and D3 (thin solid).

seemingly skillful simulation is an example where error growth has been limited artificially owing to the proximity of the analyses-forced eastern boundary to the vortex track. However, the next cyclone track to be discussed shows how distance from the eastern boundary of the respective domains affects the modeled vortex.

\section{c. Fabriola}

The modeled tracks of the cyclonelike vortex associated with Tropical Cyclone Fabriola are shown in Fig. 6 . The origin of the cyclone was inside D2 but outside D3. It subsequently moved westward into D3. The vortex simulated using D2 (eastern boundary east of the initial vortex) only reaches the east coast of Madagascar (Fig. 6b). The simulation done with D3, however, shows an improved track, which, in agreement with the analyses, has the vortex migrating across northern Madagascar into the Mozambique Channel and then across southern Madagascar before it dies in the ocean south of the island on 9 January (Fig. 6b). In the larger domain, with the eastern boundary a greater distance from Madagascar, the simulated vortex dissipates over the landmass. The smaller domain constrains the solution more closely to the driving fields, and thus the proximity to the eastern boundary in D3 permits an improved simulation of the track. The migration of vortices into the Mozambique Channel is therefore another example where the regional model can simulate tracks inside the channel as it moves across the eastern boundary and into the domain.

Atmospheric characteristics of the analyses and simulated vortices are shown in Fig. 7 (average values 
(a)

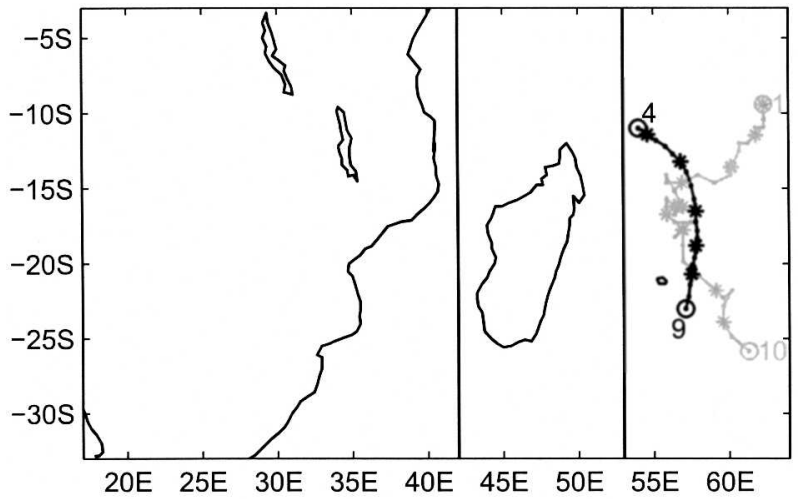

(b)

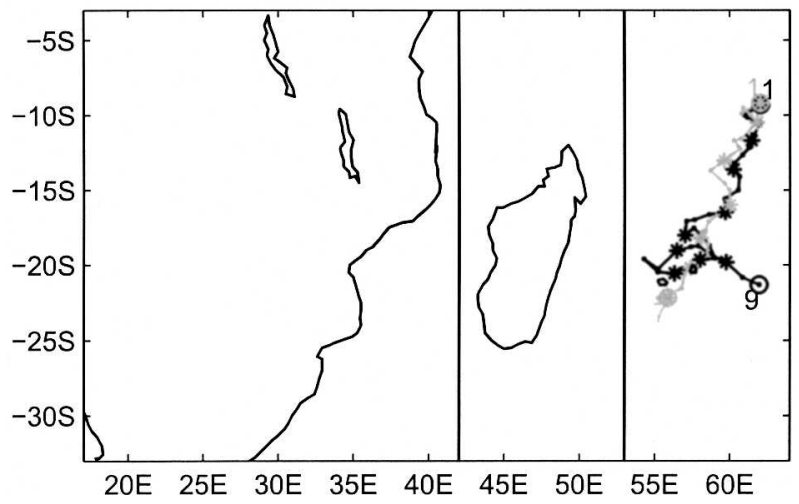

FIG. 4. (a) Observed (JTWC; black line) and ECMWF analyses (gray line) tracks of Tropical Cyclone Christelle (1-10 Jan 1995). (b) Simulated track of Christelle using domain configurations D1 (black line) and D2 (gray line). The first and last days of the storms are numbered and circled, and the tracks every $6 \mathrm{~h}$ and for each new day are marked with dots and asterisks, respectively.

around the calculated storm center). The simulation of the geopotential heights with D3 shows that the lowlevel heights of the simulated vortex deviate from the analyses after only a few model days but improve again toward the end of the modeled vortex's life cycle as the vortex curves back toward the eastern boundary. In addition, the vertical wind shear is in close agreement with that of the analyses tropical cyclone throughout its duration, as are the wind magnitudes (not shown). The vertical wind shear shown in Fig. 7 further illustrates the agreement between the modeled and analyses storm intensification using D3. The good performance of the regional model's low-level simulation at the beginning and end of its life cycle might, as above, again be attributed to the relative close proximity of the eastern boundary of D3 (Chouinard et al. 1994). However, the influence of the eastern boundary (D3) here is of a much lesser extent than the case of Christelle above, since the modeled vortex has its track well beyond the eastern boundary buffer zone (see section 2a). Therefore, the migration of the tropical cyclone-like vortex into the channel and back across Madagascar should not be seen as a consequence of the position of the
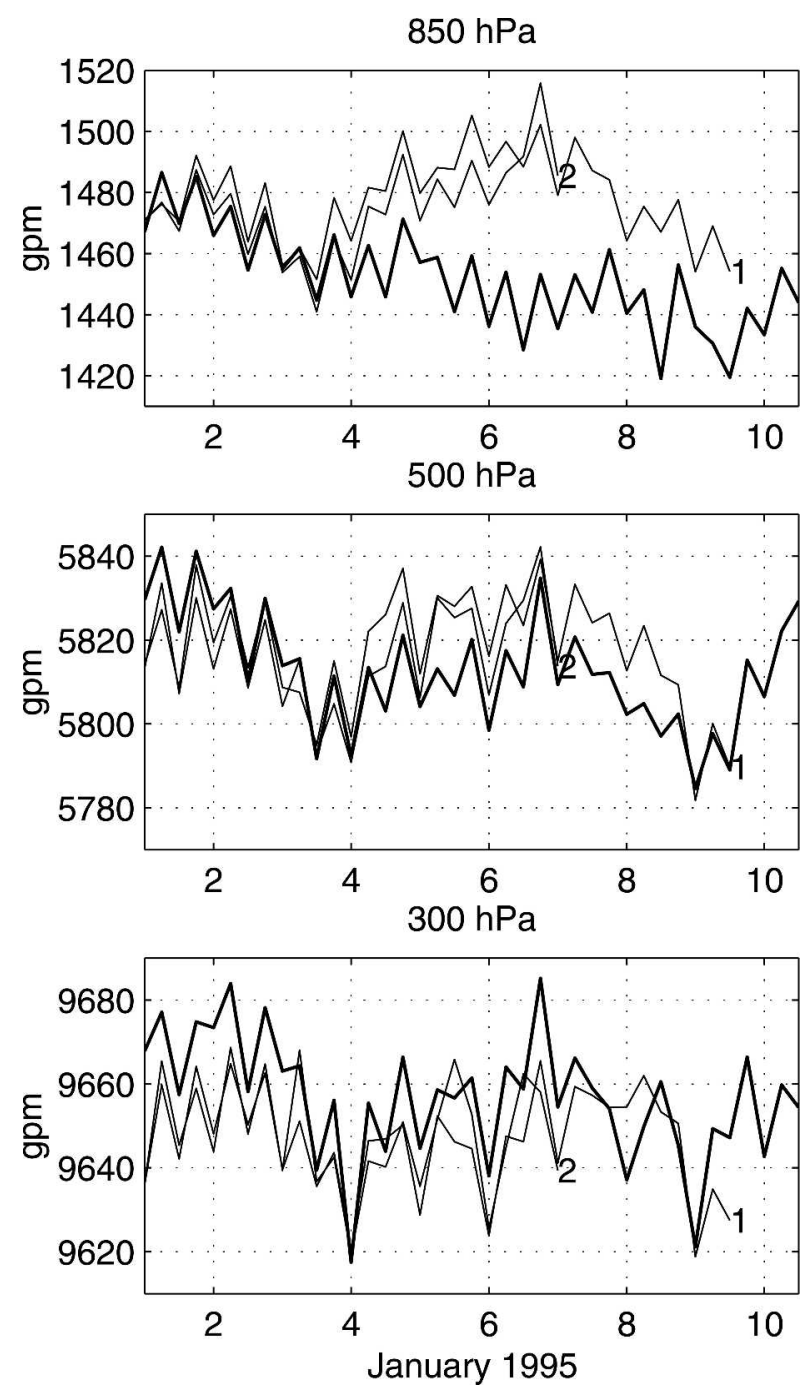

FIG. 5. Geopotential heights at 850,500 , and $300 \mathrm{hPa}$ of the analyses (thick line) and simulations (numbered thin lines) of Christelle for domain configurations D1 and D2. The thin-line numbers correspond to the domain numbers.

eastern boundary of D3, but rather of the ability of the regional model to simulate tropical cyclone-like vortex tracks over the southwestern Indian Ocean, provided that, as is found here, the storm is allowed to progress through the eastern boundary of the domain. Tropical Cyclone Gretelle, which originated close to where Fabriola had its origin, had its observed track going even farther beyond the eastern boundaries of domain configurations D2 or D3. Its associated cyclonelike vortex is discussed in greater detail next.

\section{d. Gretelle}

The modeled track of the cyclonelike vortex associated with Tropical Cyclone Gretelle is shown in Fig. 8. The analyses tropical cyclone initially developed, like Fabriola, inside D2 but outside D3. Only the simulation 
(a)

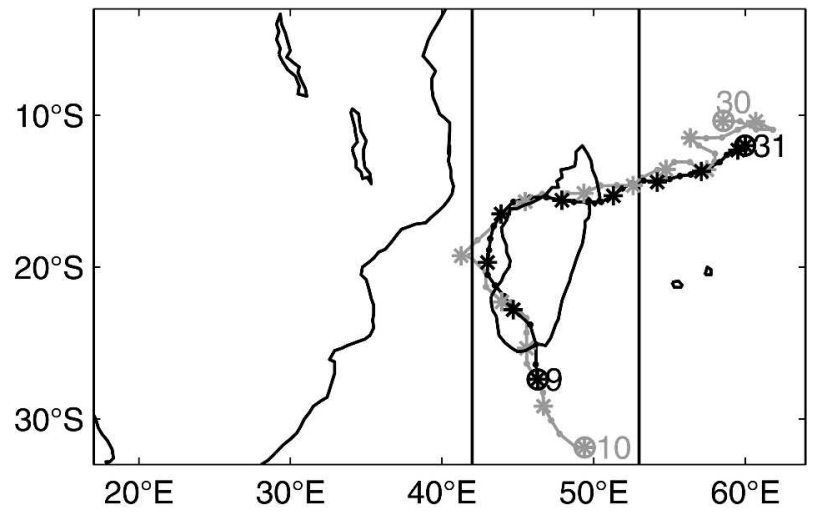

(b)

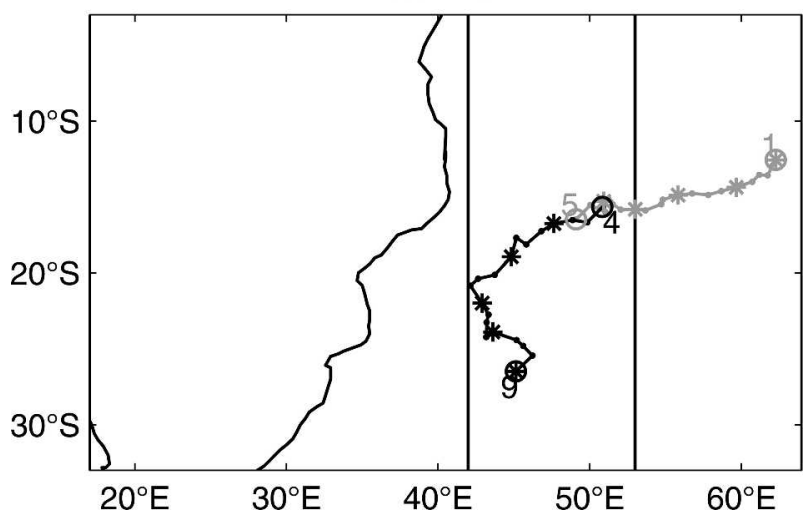

FIG. 6. (a) Observed (JTWC; black line) and ECMWF analyses (gray line) tracks of Tropical Cyclone Fabriola (31 Dec 1996 to 9 Jan 1997). (b) Simulated tracks of Fabriola using domain configurations D2 (gray line) and D3 (black line). The first and last days of the storms are numbered and circled, and the tracks every $6 \mathrm{~h}$ and for each new day are marked with dots and asterisks, respectively.

with the vortex moving through the eastern boundary of $\mathrm{D} 3$ produces a vortex life cycle duration close to that of the analyses cyclone. However, the simulated track curves to the north and results in landfall in northern Mozambique, whereas the analyses curve southward. A possible explanation for the northward curvature is the simulation of a stationary additional vortex close to the southern boundary that developed independently of the simulated Gretelle vortex, subsequently forcing the simulated track northward. Notwithstanding the cyclone's northward track, the progression of the vortex into the Mozambique Channel is again an example where vortex tracks simulated by the regional model move well beyond Madagascar. However, the simulated vortex of D3 correctly shows an initial intensification of the vortex but fails to intensify the vortex further (not shown), as was also seen with most of the cases above. These failures of a regional model to skillfully intensify simulated vortices have also been found by others (Walsh and Watterson 1997).
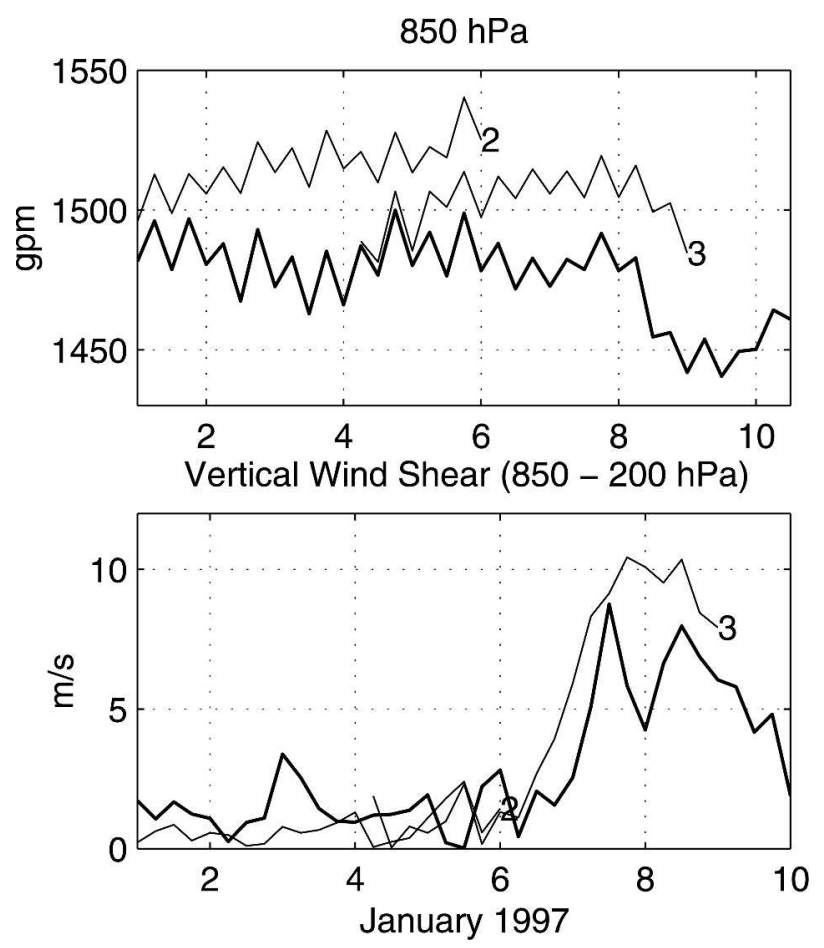

FIG. 7. Geopotential heights at $850 \mathrm{hPa}$ and vertical wind shear of the analyses (thick line) and simulations (numbered thin lines) of Fabriola for domain configurations D2 and D3. The thin-line numbers correspond to the domain numbers.

\section{e. Channel vortex simulation}

It has been shown here that the RegCM2 can simulate tropical cyclone-like vortex tracks going into the Mozambique Channel, albeit with lower vortex intensity than analyses storms. Next it is investigated if the regional model can also generate a cyclonelike vortex inside this channel on its own, since it often happens that tropical cyclones develop there (e.g., Fodah, 19-27 January 1995; Josta, 4-12 March 1995; Beltane, 12-18 February 1998; Alda, 14-19 January 1999; Dera, 9-13 March 2001; and Cyprien, 1-2 January 2002). The regional model run for the January 1995 period using D2 does simulate a weak cyclonelike vortex in the channel between southern Africa and Madagascar. Figure 9 shows the simulated 850 -hPa-height field at 0000 UTC on 21 January 1995 . This vortex agrees with the location and timing of a tropical cyclone in the analyses but is weaker than the analyses storm (not shown). However, the selection of the domain size seems to be of importance here, since the simulation with the largest domain (D1) does not show a cyclonelike vortex in the channel during the corresponding period. The fact that the largest domain does not demonstrate the cyclonic genesis seems to suggest that boundary forcing constrains the solution in the smaller domain and that the regional model physics was not sufficient to generate this cyclone on its own in the larger domain. It is quite possible that, in a similar long simulation, even a model 
(a)

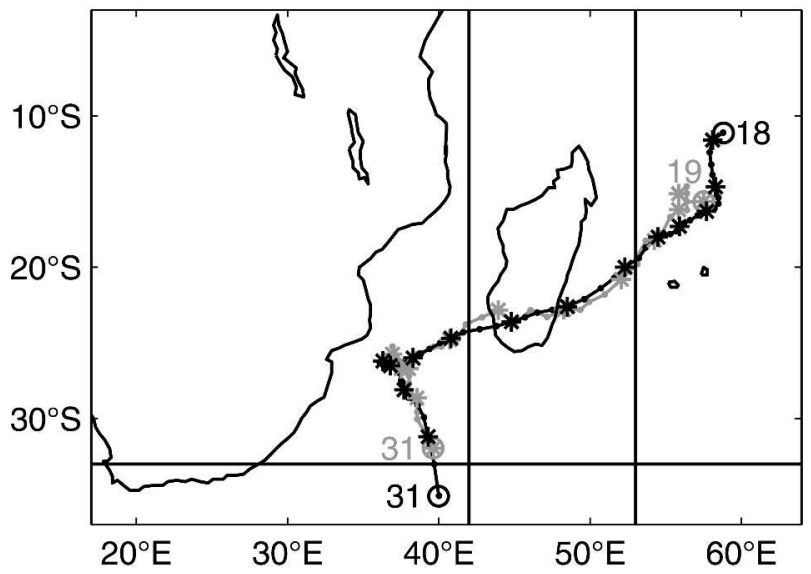

(b)

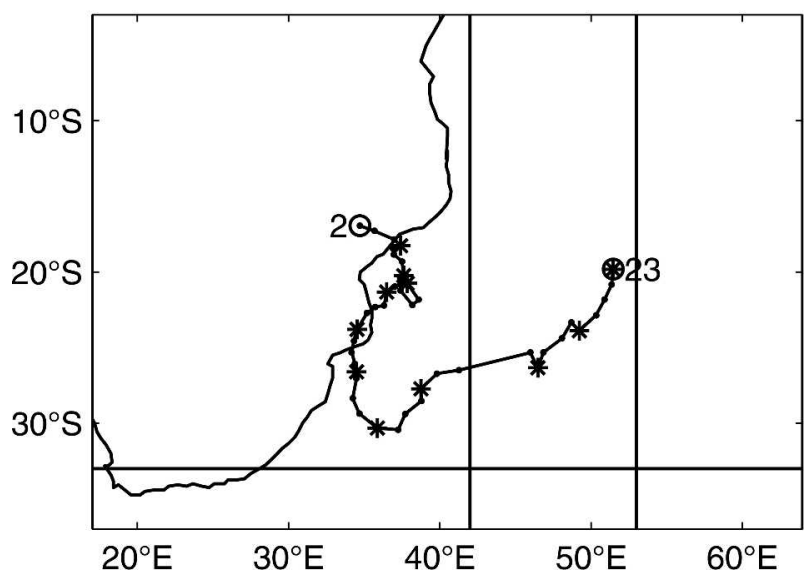

FIG. 8. (a) Observed (JTWC; black line) and ECMWF analyses (gray line) tracks of Tropical Cyclone Gretelle (19-31 Jan 1997).

(b) Simulated track of Gretelle using domain configuration D3. The first and last days of the storms are numbered and circled, and the tracks every $6 \mathrm{~h}$ and for each new day are marked with dots and asterisks, respectively.

with perfect physics would fail to generate this storm in the larger domain, because of the limitations imposed by dynamical chaos. However, one simulation using the larger domain and failing to produce the vortex is insufficient proof to conclude that this domain cannot produce vortices in the channel. This issue would require analysis of the regional model's internal variability by performing a number of simulations with perturbed boundary forcing.

\section{Discussion and conclusions}

Fine-resolution GCMs are expected to improve seasonal forecasts of tropical cyclone statistics (Vitart et al. 2003). High-resolution GCM-generated tropical cyclones have been found to produce a storm frequency and distribution that for all ocean basins represented climatology fairly realistically (Bengtsson et al. 1995). With a GCM resolution close to $2^{\circ}$, however, the mean simulated tropical storm frequency is only about half of the number observed (Vitart and Stockdale 2001). In addition, GCMs tend to simulate tropical cyclone tracks over the southern Indian Ocean too far to the east, making useful seasonal probability forecasts of tropical cyclone occurrence in the Mozambique Channel difficult when using only raw GCM output. Although regional models have been found to produce tropical cyclone-like vortices weaker than those observed, these models can produce more realistic vortices than the GCMs (Walsh and Watterson 1997). In this paper, the potential of a regional climate model over the southwestern Indian Ocean has been investigated by driving the model, RegCM2, with time-dependent large-scale meteorological analyses data. The ability of this nested scheme to simulate vortex tracks into the channel and also the development of these vortices in the channel, has been examined. Results suggest that there are potential benefits in using regional climate models to simulate tropical cyclone-like vortex tracks and their development over the southwestern Indian Ocean.

Results presented here have shown that the regional model has the ability to simulate tropical cyclone-like vortex tracks across Madagascar and into the Mozambique Channel in domains that exhibit some control from the eastern boundary. It has also been shown that Madagascar has influence over storms migrating across it and into the channel since it affects the low-level flow. However, it remains to be seen whether or not the nesting of a regional model into GCM fields will produce similar results since the regional model's ability to simulate tracks west of the island may be dependent on the GCM's ability to skillfully simulate the large-scale circulation over the southern Indian Ocean (Vitart and Stockdale 2001), as well as the simulation of tropical cyclone-like vortices east of Madagascar. Even if these vortices are skillfully simulated by the GCM, the regional model has difficulty in creating intense vortices at lower atmospheric levels. For the most part, the intensity of the cyclonelike vortices is only simulated well close to the eastern boundary of the model domain but becomes weak as the simulated vortex progresses in its westward-moving life cycle. Low-level core temperatures are also simulated to be too cold, which will subsequently hamper deep convection and might be a reason for the weak low-level vortex intensities. In addition, modeled vortices tend to move more slowly than their associated analyses storms, especially when they are moving across Madagascar.

Since it has been shown here that the regional model's potential to simulate tracks far westward depends on whether the cyclonelike vortex is allowed to move through the eastern boundary of the model domain, the correct placement of the eastern boundary in the nesting is therefore of the utmost importance. To optimize 


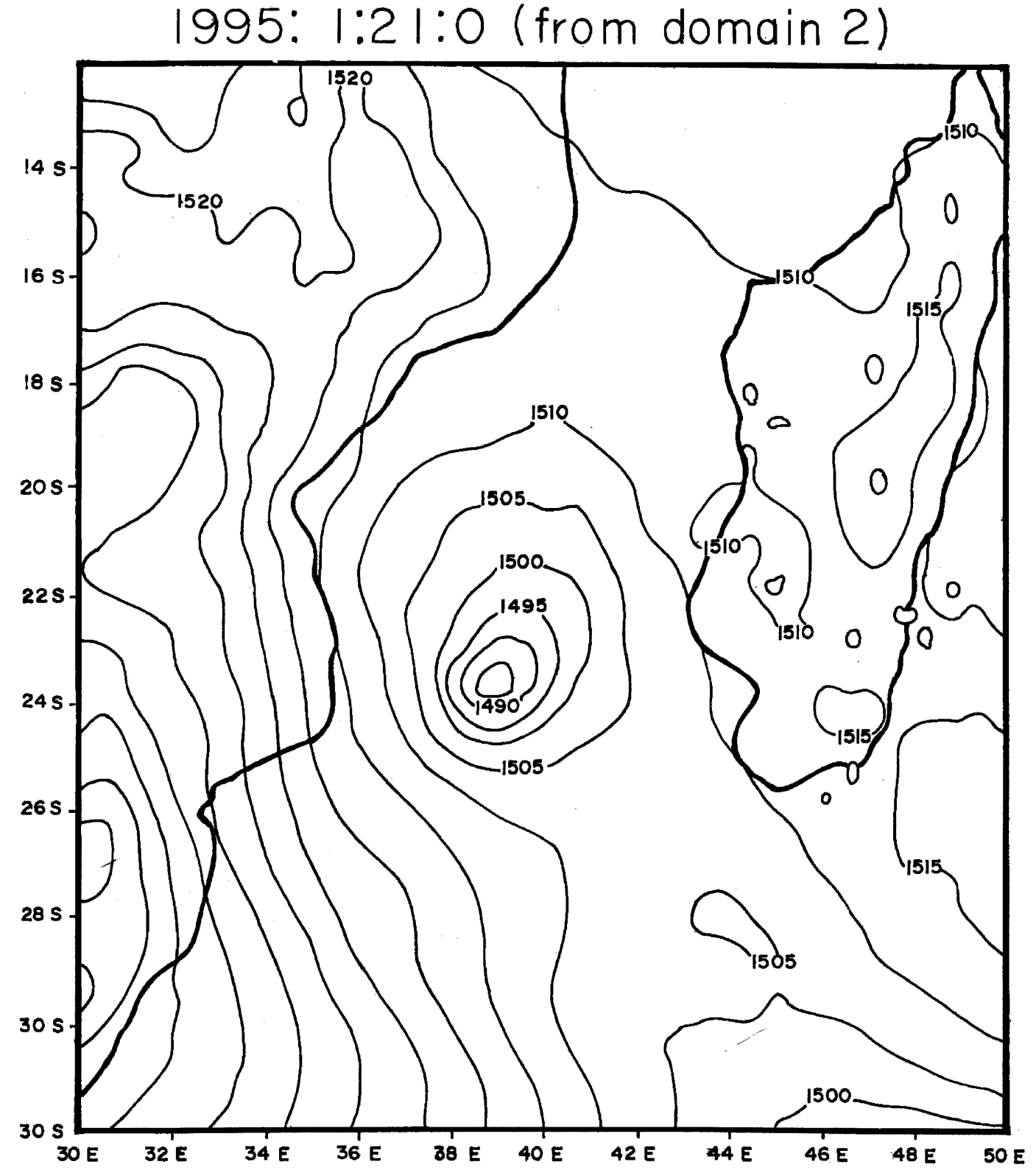

FIG. 9. Simulated 850-hPa geopotential height field of a tropical cyclone-like vortex in the Mozambique Channel using domain configuration D2.

the nesting of a regional model into the GCM largescale fields, the GCM's tropical cyclone-like vortex climatology should be investigated to determine the area east of Madagascar of highest simulated track density and the eastern boundary of the regional model should be placed across that area. The effective location of the eastern boundary is likely to be not too far east of Madagascar. The island must be included in the regional model's domain, since it affects the low-level flow and ultimately the tropical vortices' evolution over the southwestern Indian Ocean. In addition, the placement of the northern boundary has been found to affect the development of these tropical vortices in the channel. A very large domain might be less successful in channel vortex simulation than a domain with a more southward-placed northern boundary. Choosing an optimal domain to assess storm development in the channel will require ensembles of simulations driven by multiple GCM realizations, which show vortices in this region. Such a large ensemble would also help to address the unanswered question of to what extent the nested system is actually simulating tropical cyclone genesis versus just responding to information being advected in from the domain boundaries. 
Given these caveats, careful consideration of domain choice, and the quality of GCM large-scale fields, the results here suggest that the nested approach may provide useful information regarding vortices with landfall in southern Africa. The results here are sufficiently encouraging to warrant further study of the regional model nested in a GCM.

Acknowledgments. This postdoctoral research, administered by the University Corporation for Atmospheric Research, was supported by Cooperative Agreement NAO7GP0213 between the National Oceanic and Atmospheric Administration and Columbia University. Computing resources were supplied by the International Research Institute for Climate Prediction. The authors are grateful to Dr. Filippo Giorgi (ICTP), developer of the RegCM2. Also appreciated are the comments of the three anonymous referees of the paper, whose comments helped to strengthen and clarify the manuscript.

\section{REFERENCES}

Anthes, R. A., Y.-H. Kuo, E.-Y. Hsie, S. Low-Nam, and T. W. Bettge, 1989: Estimation of skill and uncertainty in regional numerical models. Quart. J. Roy. Meteor. Soc., 115, 763-806.

Barnston, A. G., S. J. Mason, L. Goddard, D. G. DeWitt, and S. E. Zebiak, 2003: Multimodel ensembling in seasonal climate forecasting at IRI. Bull. Amer. Meteor. Soc., 84, 1783-1796.

Bengtsson, L., M. Botzet, and M. Esch, 1995: Hurricane-type vortices in a general circulation model. Tellus, 47A, 175-196.

Bhaskaran, B., R. G. Jones, J. M. Murphy, and M. Noguer, 1996: Simulations of the Indian summer monsoon using a nested regional climate model: Domain size experiment. Climate Dyn., 12, 573-587.

Bormann, N., and C. J. Marks, 1999: Mesoscale rainfall forecasts over New Zealand during SALPEX96: Characterization and sensitivity studies. Mon. Wea. Rev., 127, 2880-2893.

Camargo, S. J., and S. E. Zebiak, 2002a: Variability of tropical storms in AGCMs. Preprints, 25th Conf. on Hurricanes and Tropical Meteorology, San Diego, CA, Amer. Meteor. Soc., 148-149.

— , and — 2002b: Improving the detection and tracking of tropical cyclones in atmospheric general circulation models. Wea. Forecasting, 17, 1152-1162.

— atmospheric general circulation model. Tellus, 56A, 56-67.

Chouinard, C., J. Mailhot, H. L. Mitchell, A. Staniforth, and T. Hogue, 1994: The Canadian regional data assimilation system: Operational and research applications. Mon. Wea. Rev., 122, 1306-1325.

Giorgi, F., M. R. Marinucci, and G. T. Bates, 1993a: Development of a second-generation regional climate model (RegCM2). Part I: Boundary-layer and radiative transfer processes. Mon. Wea. Rev., 121, 2794-2813.

$\longrightarrow,-\longrightarrow$, , and G. De Canio, 1993b: Development of a second-generation regional climate model (RegCM2). Part II: Convective processes and assimilation of lateral boundary conditions. Mon. Wea. Rev., 121, 2814-2832.

— C. C. S. Brodeur, and G. T. Bates, 1994: Regional climate change scenarios over the United States produced with a nested regional climate model. J. Climate, 7, 375-399.

Grell, G. A., 1993: Prognostic evaluation of assumptions used by cumulus parameterizations. Mon. Wea. Rev., 121, 764-787.

Holton, J. R., 2004: An Introduction to Dynamic Meteorology. 4th ed. Academic Press, 535 pp.

Jones, R. G., J. M. Murphy, and M. Nogeur, 1995: Simulation of climate change over Europe using a nested regional-climate model: I: Assessment of control climate, including sensitivity to location of lateral boundaries. Quart. J. Roy. Meteor. Soc., 121, 1413-1449.

Keen, T. R., and S. M. Glenn, 1998: Factors influencing model skill for hindcasting shallow water currents during Hurricane Andrew. J. Atmos. Oceanic Technol., 15, 221-236.

Kurihara, Y., M. A. Bender, and R. J. Ross, 1993: An initialization scheme for hurricane models by vortex specification. Mon. Wea. Rev., 121, 2030-2045.

Liu, Y., D.-L. Zhang, and M. K. Yau, 1997: A multiscale numerical study of Hurricane Andrew (1992). Part I: Explicit simulation and verification. Mon. Wea. Rev., 125, 3073-3093.

McGregor, J. L., 1997: Regional climate modeling. Meteor. Atmos. Phys., 63, 105-117.

Nguyen, K. C., and K. J. E. Walsh, 2001: Interannual, decadal, and transient greenhouse simulation of tropical cyclone-like vortices in a regional climate model of the South Pacific. J. Climate, 14, 3043-3054.

Peng, M. S., B.-F. Jeng, and C.-P. Chang, 1993: Forecast of typhoon motion in the vicinity of Taiwan during 1989-90 using a dynamical model. Wea. Forecasting, 8, 309-325.

Powell, M. D., and S. D. Aberson, 2001: Accuracy of United States tropical cyclone landfall forecasts in the Atlantic basin. Bull. Amer. Meteor. Soc., 82, 2749-2767.

Reynolds, R. W., and T. M. Smith, 1994: Improved global sea surface temperature analyses using optimum interpolation. $J$. Climate, 7, 929-948.

Serrano, E., 1997: Tropical cyclones. ECMWF Re-Analysis Project Report Series 5, European Centre for Medium-Range Weather Forecasts, Reading, United Kingdom, 30 pp.

_ and P. Unden, 1994: Evaluation of a tropical cyclone bogusing method in data assimilation and forecasting. Mon. Wea. Rev., 122, 1523-1547.

Seth, A., and F. Giorgi, 1998: The effects of domain choice in summer precipitation simulation and sensitivity in a regional climate model. J. Climate, 11, 2698-2712.

_ R. C. Bales, and R. E. Dickinson, 1999: A framework for the study of seasonal snow hydrology and its interannual variability in the alpine regions of the Southwest. J. Geophys. Res., 104, 22 117-22 135.

Trenberth, K. E., 1992: Global analyses from ECMWF and atlas of 1000 to $10 \mathrm{mb}$ circulation statistics. NCAR Tech. Note NCAR/TN-373+STR, 191 pp.

Ueno, M., 1989: Operational bogussing and prediction of typhoon in JMA. JMA/NPD Tech. Rep. 28, 49 pp. [Available from Japan Meteorological Agency/Numerical Prediction Division, Ote-machi 1-3-4, Chiyoda-ku, Tokyo 100, Japan.]

Vitart, F., and T. N. Stockdale, 2001: Seasonal forecasting of tropical storms using coupled GCM integrations. Mon. Wea. Rev., 129, 2521-2537.

- J. L. Anderson, and W. F. Stern, 1997: Simulation of interannual variability of tropical storm frequency in an ensemble of GCM integrations. J. Climate, 10, 745-760.

$\ldots, \ldots$, and _ 1999: Impact of large-scale circulation and tropical storm frequency, intensity, and location, simulated by an ensemble of GCM integrations. J. Climate, 12, 32373254.

_, D. Anderson, and T. N. Stockdale, 2003: Seasonal forecasting of tropical cyclone landfall over Mozambique. J. Climate, 16, 3932-3945.

Walsh, K., 1997: Objective detection of tropical cyclones in highresolution analyses. Mon. Wea. Rev., 125, 1767-1779.

_ , and L. McGregor, 1995: January and July climate simulations over the Australian region using a limited-area model. J. Climate, 8, 2387-2403.

_ , and I. G. Watterson, 1997: Tropical cyclone-like vortices in a limited area model: Comparison with observed climatology. J. Climate, 10, 2240-2259. 